\title{
Increased circulating choline, L-carnitine and TMAO levels are related to changes in adiposity during weight loss: role of the gut microbiota?
}

\author{
Ellen E. Blaak, Emanuel E. Canfora \\ Department of Human Biology, NUTRIM School of Nutrition and Translational Research in Metabolism, Maastricht University Medical Centre+, \\ Maastricht, The Netherlands \\ Correspondence to: Emanuel E. Canfora, PhD. Department of Human Biology, NUTRIM School of Nutrition and Translational Research in \\ Metabolism, Maastricht University Medical Centre+, Universiteitssingel 50, 6229 ER, Maastricht, PO Box 616, 6200 MD, Maastricht, The \\ Netherlands. Email: Emanuel.canfora@maastrichtuniversity.nl. \\ Provenance: This is an invited Editorial commissioned by the Section Editor Jianqing Tian (Department of Endocrinology, Xiamen Humanity \\ Hospital, Xiamen, China). \\ Comment on: Heianza Y, Sun D, Smith SR, et al. Changes in Gut Microbiota-Related Metabolites and Long-term Successful Weight Loss in \\ Response to Weight-Loss Diets: The POUNDS Lost Trial. Diabetes Care 2018;41:413-9.
}

Submitted Nov 01, 2018. Accepted for publication Nov 05, 2018.

doi: $10.21037 / \mathrm{atm} .2018 .11 .11$

View this article at: http://dx.doi.org/10.21037/atm.2018.11.11

The gut microbiome has been increasingly recognized as contributor to the development of obesity and associated disorders such as cardiovascular disease, type 2 diabetes mellitus and several forms of cancers $(1,2)$. Techniques to assess fecal microbial composition rapidly developed in the last decade, which provide us important insight on how gut microbes are involved in these metabolic disorders and diseases. However, as genomic approaches transform our understanding of microbial species in health and disease, many of their functions and phenotypes are currently unknown. Therefore, it is important that research in humans focuses not only on microbial composition but also on its activity by assessing microbial products in i.e., feces, plasma or urine. Microbial metabolites derived from fermentation of indigestible carbohydrates such as shortchain fatty acids and succinate are extensively studied and identified as important regulators of the host's substrate and energy metabolism (3-5). Besides fermentation of indigestible carbohydrates, gut microbes are able to convert dietary choline and L-carnitine via microbial enzymes into dimethylamine (DMA) and trimethylamine (TMA), thereby affecting circulating choline and L-carnitine. DMA and TMA can be transported via the portal vein to the liver where they can be further metabolized into trimethylamine $\mathrm{N}$-oxide (TMAO). Evidence for the fundamental role of the microbiota in TMA production is derived from germfree mice, which do not excrete TMA (6). Previous studies reported that TMAO production is altered in individuals with obesity, insulin resistance and type 2 diabetes mellitus $(7,8)$. In the Diabetes Care publication of January 2018, Heianza et al. [2018] (9) investigated the effect of 6-month diet-induced weight loss on TMAO, choline and L-carnitine plasma concentrations and how they are related to changes in body weight, overall and central adiposity, body composition and fat distribution in a large and well controlled cohort of 510 overweight and obese adults (the POUNDS lost trial). In addition, they investigated whether early changes in these metabolites levels are predictable for successful longer-term weight loss.

The research team firstly demonstrated in a crosssectional analysis that circulating plasma concentrations of TMAO, choline and L-carnitine are positively associated with body-mass-index, body fat composition and visceral fat mass in overweight and obese individuals without diabetes. Next, they showed that individuals with greater reduction in plasma choline or L-carnitine concentrations, rather than in concentrations of TMAO itself, achieved greater reduction in body weight at 6-month after the start of the low-calorie weight-loss diet. In addition, the study's results indicated an inverse relationship between changes in plasma 
TMAO and choline concentrations and changes in wholebody fat mass and fat distribution after the 6-month weight loss diet. Of interest, the changes in choline and L-carnitine concentrations after 6-month were predictive of long-term ( 2 years follow up) improvements in adiposity and weight loss (9). These findings are of clinical relevance, since improvements in body weight loss and adiposity markers are strongly related to alleviations in cardiometabolic diseases (10). Of note, the same research group demonstrated in another publication using a similar cohort of the POUNDS lost trial (11) that reductions in choline and TMAO plasma concentrations were associated with greater improvements in markers of insulin sensitivity and glucose homeostasis after the 6-months weight-loss diet. In addition, changes in circulating choline concentrations were associated to longterm improvements in glucose and insulin metabolism (11). Therefore, their findings suggest that circulating L-carnitine, choline and TMAO concentrations could be interesting biomarkers for successful reductions in body weight and cardiometabolic risk factors in diet-induced weight loss interventions. Furthermore, the report's outcomes suggest that nutritional or pharmacological strategies to decrease circulating TMAO, choline and L-carnitine levels might be of interest to prevent or treat metabolic disorders and diseases. In agreement with these findings, a recent publication combining animal and human studies demonstrated that mice lacking a host enzyme called flavin monooxygenase (FMO) 3, which converts TMAO into its active form, are protected against high-fat dietinduced adiposity and weight gain. In addition, the report showed that high levels of TMAO were associated with higher incidences of type 2 diabetes mellitus (12).

A logical nutritional strategy may be the reduction of foods that contain high amounts of choline and L-carnitine such as red meats and eggs in your daily diet. Indeed, studies in mice on a choline-deficient diet showed a significant weight loss (13) and improvements in insulin sensitivity and glucose tolerance (14). Additionally, increased circulating L-carnitine concentrations have been positively associated with circulating glucose concentrations in women (15). In contrast, however, clinical trials indicated that L-carnitine supplementation led to improvements in glycemic control in type 2 diabetic individuals (16). Another nutritional strategy may be the supplementation of soluble fibers. Animal and human data showed that increased soluble fiber intake reduce TMA/TMAO metabolism, and that this was associated with increased SCFA production and reduced circulating lipids and cholesterol levels $(17,18)$.
The reported alterations in choline, L-carnitine and TMAO metabolism may be mediated by the gut microbiota. Therefore, alterations in gut microbial metabolite production might be predictive for success in weight loss and cardiometabolic risk markers. In accordance, in an recently published study (19) faecal metagenome analysis was combined with the hepatic transcriptome and plasma and urine metabolomes analysis in morbidly obese women. Here, the status of hepatic steatosis was associated with decreased gut microbial gene richness and dysregulation of TMA/TMAO metabolism. These observations identified the TMA/TMAO metabolism as important contributor to the development of metabolic disorders. However, it should be noted that in the latter study also other dysbiosis associated factors, such as branched-chain amino acids, proteolytic fermentation products and lipopolysaccharides were identified as important contributors to hepatic steatosis. Nevertheless, based on mainly animal experiments several direct pathogenic mechanism can be postulated to link increased microbial TMA/ TMAO metabolism to cardiometabolic diseases. These include disturbances in host sterol and lipid metabolic pathways (20), activation of the NLRP3 inflammasome (21) and nuclear factor-kappa pathway thereby possibly contributing to lowgrade inflammation in the obese metabolically disturbed phenotype (22).

A limitation of the study of Heianza et al. [2018] (9) is that fecal microbiota samples were not available. Thus, no direct relationship between diet-induced alterations in gut microbiota composition or functionality with an altered TMAO metabolism was assessable. Therefore, it is important to mention that different pathways can be involved in the production of TMAO. Next to the involvement of microbial metabolites in its biosynthesis, TMAO can be derived from endogenous sources involving host hepatic FMO or directly from exogenous sources that contain TMAO such as fish and other seafoods (23). In addition, dietary fat intake might influence circulating TMAO levels, since in a study with non-obese individuals increased postprandial plasma TMAO concentrations were found 4 hours after a high-fat meal (24), which suggests microbial-independent mechanisms. Furthermore, the alteration in circulating choline and L-carnitine level does not per se reflect changes in the microbial metabolism after diet-induced weight loss, but might be related to a misalignments in absorption, tissue uptake or host metabolism in metabolically disturbed individuals and might be influenced by other endogenous and exogenous nutrient sources. 
In conclusion, the interesting study by Heianza et al. [2018] provides evidence that the diet-derived metabolites choline and L-carnitine and its related metabolite TMAO are involved in the success of weight loss and improvement in adiposity and body composition. Although the exact mechanisms remain to be elucidated, this study provides evidence that the choline/TMAO and L-carnitine/TMAO metabolism could be targets and/or biomarkers for weight loss success. Further studies have to elucidate the role of microbial choline and L-carnitine metabolism in these processes, since neither the microbial composition nor its functionality was assessed in this study. As described above TMAO can also be derived from exogenous sources and endogenic processes and as demonstrated previously these processes are increased in patients with obesity, type 2 diabetes mellitus and hepatic steatosis. Moreover, the differences in choline and L-carnitine concentrations do not ultimately reflect changes in the microbiome, but might be caused by other exogenous and endogenous nutrient sources and misalignments in metabolic processes dependent on the host cardiometabolic phenotype. In addition, recent large-scale observational studies indicate that besides choline and TMA/TMAO metabolism, several microbial derived metabolites such as short-chain fatty acids, lipopolysaccharides, branched-chain amino acids and other proteolytic products are involved in the progression of NAFLD, insulin resistance and type 2 diabetes mellitus. This further emphasize that multifactorial processes related to the microbiome are involved in metabolic diseases. However, to our knowledge a role of weight-loss induced chances in these metabolites, a direct link with alterations in gut microbiota composition and its functionality and changes in the host metabolic phenotype is currently not available. Therefore, combining interventions with deep phenotypically assessments of the individual with new approaches to measure microbial functionality such as metagenomics, metabolomics or metatranscriptomics can provide new insight in the relationship of the microbiome and host metabolic diseases.

\section{Acknowledgements}

None.

\section{Footnote}

Conflicts of Interest: The authors have no conflicts of interest to declare.

\section{References}

1. Cho I, Blaser MJ. The human microbiome: at the interface of health and disease. Nat iews Genet 2012;13:260.

2. Nieuwdorp M, Gilijamse PW, Pai N, et al. Role of the microbiome in energy regulation and metabolism. Gastroenterology 2014;146:1525-33.

3. Canfora EE, Blaak EE. Acetate: a diet-derived key metabolite in energy metabolism: good or bad in context of obesity and glucose homeostasis? Curr Opin Clin Nutr Metab Care 2017;20:477-83.

4. de Vadder F, Mithieux G. Gut-brain signaling in energy homeostasis: the unexpected role of microbiota-derived succinate. J Endocrinol 2018;236:R105-8.

5. Canfora EE, Jocken JW, Blaak EE. Short-chain fatty acids in control of body weight and insulin sensitivity. Nat Rev Endocrinol 2015;11:577-91.

6. Al-Waiz M, Mikov M, Mitchell S, et al. The exogenous origin of trimethylamine in the mouse. Metabolism 1992;41:135-6.

7. Dambrova M, Latkovskis G, Kuka J, et al. Diabetes is associated with higher trimethylamine $\mathrm{N}$-oxide plasma levels. Exp Clin Endocrinol Diabetes 2016;124:251-6.

8. Lever M, George PM, Slow S, et al. Betaine and trimethylamine- $\mathrm{N}$-oxide as predictors of cardiovascular outcomes show different patterns in diabetes mellitus: an observational study. PloS One 2014;9:e114969.

9. Heianza Y, Sun D, Smith SR, et al. Changes in Gut Microbiota-Related Metabolites and Long-term Successful Weight Loss in Response to Weight-Loss Diets: The POUNDS Lost Trial. Diabetes Care 2018;41:413-9.

10. Goodpaster BH, DeLany JP, Otto AD, et al. Effects of diet and physical activity interventions on weight loss and cardiometabolic risk factors in severely obese adults: a randomized trial. JAMA 2010;304:1795-802.

11. Heianza Y, Sun D, Li X, et al. Gut microbiota metabolites, amino acid metabolites and improvements in insulin sensitivity and glucose metabolism: the POUNDS Lost trial. Gut 2018. [Epub ahead of print].

12. Schugar RC, Shih DM, Warrier M, et al. The TMAOproducing enzyme flavin-containing monooxygenase 3 regulates obesity and the beiging of white adipose tissue. Cell Rep 2017;19:2451-61.

13. Rizki G, Arnaboldi L, Gabrielli B, et al. Mice fed a lipogenic methionine-choline-deficient diet develop hypermetabolism coincident with hepatic suppression of SCD-1. J Lipid Res 2006;47:2280-90.

14. Raubenheimer PJ, Nyirenda MJ, Walker BR. A choline- 
deficient diet exacerbates fatty liver but attenuates insulin resistance and glucose intolerance in mice fed a high-fat diet. Diabetes 2006;55:2015-20.

15. Malinowska AM, Szwengiel A, Chmurzynska A. Dietary, anthropometric, and biochemical factors influencing plasma choline, carnitine, trimethylamine, and trimethylamine-N-oxide concentrations. Int J Food Sci Nutr 2017;68:488-95.

16. Bene J, Hadzsiev K, Melegh B. Role of carnitine and its derivatives in the development and management of type 2 diabetes. Nutr Diabetes 2018;8:8.

17. Li Q, Wu T, Liu R, et al. Soluble Dietary Fiber Reduces Trimethylamine Metabolism via Gut Microbiota and CoRegulates Host AMPK Pathways. Mol Nutr Food Res 2017;61:1700473.

18. De Filippis F, Pellegrini N, Vannini L, et al. High-level adherence to a Mediterranean diet beneficially impacts the gut microbiota and associated metabolome. Gut 2016;65:1812-21.

19. Hoyles L, Fernández-Real J-M, Federici M, et al. Molecular phenomics and metagenomics of hepatic

Cite this article as: Blaak EE, Canfora EE. Increased circulating choline, L-carnitine and TMAO levels are related to changes in adiposity during weight loss: role of the gut microbiota? Ann Transl Med 2018;6(Suppl 2):S92. doi: 10.21037/atm.2018.11.11 steatosis in non-diabetic obese women. Nat Med 2018;24:1070.

20. Koeth RA, Wang Z, Levison BS, et al. Intestinal microbiota metabolism of L-carnitine, a nutrient in red meat, promotes atherosclerosis. Nat Med 2013;19:576.

21. Yue C, Yang X, Li J, et al. Trimethylamine N-oxide prime NLRP3 inflammasome via inhibiting ATG16L1-induced autophagy in colonic epithelial cells. Biochem Biophys Res Commun 2017;490:541-51.

22. Al-Obaide MA, Singh R, Datta P, et al. Gut microbiotadependent trimethylamine-N-oxide and serum biomarkers in patients with T2DM and advanced CKD. J Clin Med 2017;6:86.

23. Cho CE, Taesuwan S, Malysheva OV, et al. Trimethylamine-N-oxide (TMAO) response to animal source foods varies among healthy young men and is influenced by their gut microbiota composition: A randomized controlled trial. Mol Nutr Food Res 2017;61.

24. Boutagy NE, Neilson AP, Osterberg KL, et al. Short-term high-fat diet increases postprandial trimethylamine-Noxide in humans. Nutr Res 2015;35:858-64. 